\title{
Expecting the worst? The relationship between retrospective and prospective appraisals of illness on quality of life in prostate cancer survivors
}

\author{
Rebecca Maguire $^{1}$ (D) । Paul Hanly ${ }^{2}$ (D) | Frances J. Drummond ${ }^{3}$ (D) । Anna Gavin ${ }^{4}$ (D) । \\ Linda Sharp 5 (D)
}

${ }^{1}$ Maynooth University, Maynooth, Ireland

${ }^{2}$ National College of Ireland, Dublin, Ireland

${ }^{3}$ Cork Cancer Research Centre, University College Cork, Cork, Ireland

${ }^{4}$ Queens University Belfast, Belfast, UK

${ }^{5}$ Newcastle University, Newcastle upon Tyne, UK

\section{Correspondence}

Rebecca Maguire, Department of Psychology, Maynooth University, Maynooth, Co. Kildare, Ireland.

Email: rebecca.maguire@mu.ie

Funding information

Prostate Cancer UK, Grant/Award Numbers: NI09-03 \& NI-PG13-001, NI-PG13-001 and NI09-03; National Cancer Registry Ireland; Public Health Agency for Northern Ireland; National Cancer Control Programme; R\&D office of the Public Health Agency in Northern Ireland; Health Research Board in the Republic of Ireland, Grant/Award Number: HRA_HSR/ 2010/17

\begin{abstract}
Objective: Despite a generally good prognosis, many prostate cancer survivors have poor quality of life (QOL). A greater understanding of how psychological appraisals influence QOL is merited given their potentially modifiable nature. In this study, we considered how elements of survivors' retrospective and prospective appraisals relate to QOL.

Methods: A total of 1229 prostate cancer survivors between 2 and 5 years post-diagnosis, identified from a population-based National Cancer Registry, were asked questions on their socio-demographics, health, treatment received, and adverse-effects using a cross-sectional design. QOL was assessed using the EORTC QLQ-C30. Retrospective appraisals were assessed by asking survivors to reflect on their experience of treatment-related adverse-effects compared with their prior expectations. A fear of recurrence scale assessed prospective appraisals of future disease course. A multiple regression model explored the impact of psychological appraisals on QOL, after controlling for socio-demographic, treatment, and health-related factors.
\end{abstract}

Results: The model was significant explaining $37 \%$ of variance in QOL. The strongest associate with QOL was fear of recurrence $(\beta=-.29 ; P<.001)$. Survivors who experienced side effects that were worse than expected had significantly lower QOL ( $\beta=-.10 ; P=.002)$. Other significant correlates of lower QOL were presence of comorbidities, having undergone a less invasive treatment, and having more advanced disease. Working at diagnosis and having a higher level of education were significantly associated with higher QOL.

Conclusions: Results suggest both retrospective and prospective appraisals are independently related to QOL in prostate cancer. Providing survivors with more information about possible adverse effects of treatment, as well as providing appropriate information regarding future disease progression, may improve QOL.

\section{KEYWORDS}

adverse side effects, expectations, fear of recurrence, oncology, prostate cancer, psychological appraisals, quality of life

\section{1 | INTRODUCTION}

It is well established that a cancer diagnosis and its treatment can lead to detriments in quality of life (QOL) among survivors. ${ }^{1}$ While survival rates for prostate cancer are high and increasing, ${ }^{2}$ some survivors have low QOL. ${ }^{3}$ This can, in part, be attributed to the numerous adverseeffects that often ensue following diagnosis and treatment, including urinary incontinence, bowel problems, erectile dysfunction, and fatigue. ${ }^{4}$ Such side effects can often be debilitating with potential to cause considerable impact on survivors' daily lives and psychological wellbeing. ${ }^{5-7}$

While clinical factors such as cancer stage, time since diagnosis, comorbidities, and treatment are known to be associated with QOL in cancer survivors, ${ }^{3,6,8}$ there is a growing recognition of the important 
role that psychological appraisals and perceptions can play in survivor well-being. ${ }^{9,10}$ For example, research has shown that a survivor's expectations over future disease course, specifically their fears of recurrence (FOR), can negatively impact QOL. ${ }^{11,12}$ FOR, which can be viewed as a prospective appraisal, encapsulating survivors' fears and uncertainty about future disease progression, is a common concern among cancer survivors, ${ }^{12,13}$ including those with prostate cancer. ${ }^{14-18}$ Such fears are likely to have an impact on QOL even in the absence of a genuine clinical risk of recurrence.

Survivors also make retrospective appraisals of their illness and treatment, ${ }^{19}$ and these too could influence wellbeing. ${ }^{20}$ In a recent meta-analysis, ${ }^{21}$ survivors' expectations of side effects, or Response Expectancies, were found to be related to later experience post-treatment. In prostate cancer, many patients have unrealistic expectations of side effects. ${ }^{22}$ Therefore, if survivors experience treatment-related side effects that they had not anticipated, or more specifically, that were worse than expected, this may lead to regret over treatment decisions and, hence, lower QOL. ${ }^{23}$ However, little research has investigated whether retrospective appraisals of this nature are associated with QOL. Theories of expectation violation imply that the more discrepant an individual's experience is with their prior expectations, the greater the state of cognitive disequilibrium. ${ }^{24}$ However, it is unclear whether expectation violations resulting in positive (ie, better than expected) and negative (ie, worse than expected) outcomes would have the same effect on QOL.

This paper aims to establish whether reflections on prior treatment expectations (a retrospective appraisal) and FOR (a prospective appraisal) are independently associated with QOL in prostate cancer survivors, after controlling for known socio-demographic, health, and treatment-related predictors of QOL. A greater understanding of both retrospective and prospective appraisals is merited as this offers the potential to inform interventions aimed at increasing QOL in addition to interventions focusing on health status and the management of side effects. We hypothesise that both appraisals will have an independent relationship with QOL.

\section{2 | METHODS}

\section{1 | Ethical statement}

Prior to gathering data, ethical approval was granted from the Irish College of General Practitioners. Survivors were identified from the National Cancer Registry, Ireland which contains information on all those diagnosed with prostate cancer in the Republic of Ireland. Exclusion criteria included being deceased, under the age of 18 , less than 2 years post-diagnosis, suffering from any cognitive impairment, or not being well enough to complete the questionnaire. Any men deemed ineligible by health care professionals were also excluded.

\section{2 | Design and sample}

This study was nested within the PiCTure project which investigated the experiences and outcomes of prostate cancer survivors in Ireland. The project's objective was to perform an international populationbased Patient-Reported Outcomes study relating to the diagnosis and treatment of prostate cancer. ${ }^{5,7,25}$ The hypotheses for the current study fit with this objective. Following screening for eligibility by general practitioners, 4453 survivors were invited to complete a postal questionnaire between April and September 2012. Separate questionnaires were developed for survivors between 2 and 5 years post diagnosis and those greater than 5 years post-diagnosis. Survivors in the Republic of Ireland between 2 and 5 years post-diagnosis were asked to complete a FOR scale in addition to a core questionnaire (see supplementary figure). It is these survivors who were focused on in the current study. The 5-year post diagnosis mark is a critical milestone for survivors with the expectation of a lower risk of recurrence at this stage.

\section{3 | Measures}

\subsection{1 | Quality of life}

QOL was measured using the EORTC QLQC $30^{1}$ which has been used in prostate cancer populations. ${ }^{5}$ This scale contains 30 items which comprises 5 functional scales (physical, role, social, emotional, and cognitive functioning), 9 symptom scales (fatigue, pain, nausea, dyspnoea, insomnia, appetite loss, constipation, and financial difficulties), and an overall global health score (GHS). With the exception of the GHS, survivors are required to rate to what extent they have experienced problems in the past week in each of the 14 domains on a scale of 1 (not at all) to 4 (very much). Following recent developmental work, ${ }^{26}$ we computed a summary QOL score by calculating the average of all 5 functional domains and 8 of the 9 symptom domains (financial impact is not included in this computation, nor is the GHS). Scores were then standardised to give an overall value of between 0 and 100 with higher scores indicating better QOL. This has been shown to be a robust index of QOL, performing similarly, and in some cases superiorly, to the original underlying QLQ-C30 subscales. ${ }^{26}$

\subsection{2 | Socio-demographic and health information at diagnosis}

On the questionnaire, survivors provided information on their age, marital status, employment status at diagnosis, and whether they had, or had not experienced, any of a range of comorbidities at diagnosis (specifically, heart disease, lung disease, stroke, diabetes, depression, high blood pressure, diverticular disease, bowel problems, other cancer, or any other health problems). Information on time since diagnosis and stage at diagnosis was obtained from NCRI records.

\subsection{3 | Treatment, side effects, and retrospective appraisals}

Survivors were asked to indicate whether they had received any of the following treatments for their cancer: radical prostatectomy (RP), external beam radiotherapy (ERBT), brachytherapy (BT), and/or androgen deprivation therapy (ADT). They were also asked if they had ever undergone watchful waiting or active surveillance. Based on this information and following Drummond et $\mathrm{al}^{5}$ a single hierarchical variable was created to encapsulate treatment invasiveness: RP, ERBT with concurrent ADT, ERBT without concurrent ADT, brachytherapy, ADT alone, and monitoring only (watchful waiting and/or surveillance). 
Survivors were also asked to report if they were currently experiencing any of the following potential treatment-related side effects: urinary incontinence; bowel problems; gynecomastia; sweats; hot flashes; fatigue; loss of sexual desire; and/or depression. ${ }^{4}$ This enabled a total number of ongoing side effects to be computed ranging from 0 to 8 .

Another question required survivors to reflect on whether or not their experience of side effects was in line with what they had expected. Following Gray et al, ${ }^{27}$ they were asked to indicate whether they (1) did not experience any side effects, or had side effects that were (2) not as bad as expected, (3) the same as expected, or (4) worse than expected. This was used as a measure of retrospective appraisals of side effects with higher scores representing more negative violations of expectations.

\subsection{4 | Prospective appraisals-fear of recurrence}

FOR was measured using a 5 -item scale ${ }^{28}$ which has been previously used in prostate cancer populations. ${ }^{14,16}$ This required survivors to report their worries over cancer (eg, "I will probably relapse within the next years," "My fear of cancer getting worse gets in the way of my enjoying life") as well as 1 item relating to optimism over prognosis ("I am certain that I have been cured of cancer"). Items are scored on a 5 -point Likert scale ranging from strongly agree to strongly disagree. After recoding the optimism item, total values were summed and standardised to provide an overall score from 0 to 100 with higher scores corresponding to greater FOR. Reliability for this measure was good with a Cronbach's alpha in our sample of 0.84 .

\subsection{5 | Statistical analysis}

Variables were entered into a hierarchical multiple regression model with 2 separate blocks. The first block included socio-demographic, treatment-related, and health-related information, specifically: current survivor age, marital status, highest level of education completed, employment status at diagnosis, number of comorbidities, time since diagnosis, cancer stage, treatments received, and number of treatment-related side effects. The second block included the 2 psychological appraisals, specifically prior expectations of side effects (retrospective appraisal) and FOR (prospective appraisal). The criterion variable was QOL as measured by the summary score from the QLQC30. Descriptive statistics were calculated, and preliminary analysis undertaken to ensure that no violations regarding assumptions of normality, linearity, or homoscedasticity. Correlation analysis revealed no notable multicollinearity between the predictor variables (see supplementary table). All variables in the 2 blocks were included in the model. Associations with QOL were assessed using 2-sided t-tests and $P$ values of $<0.05$ were considered significant.

\section{3 | RESULTS}

\section{1 | Descriptive statistics}

A total of 817 survivors were included in the analysis with those missing data excluded. Table 1 displays descriptive statistics for the sample. The majority of survivors (81\%) were married/cohabiting and had
TABLE 1 Descriptive statistics

\begin{tabular}{|c|c|c|c|c|}
\hline \multicolumn{2}{|l|}{ Categorical Variables } & No. & \multicolumn{2}{|r|}{$\%$} \\
\hline \multicolumn{5}{|l|}{ Marital status } \\
\hline \multicolumn{2}{|l|}{ Married/cohabiting } & \multicolumn{2}{|l|}{658} & $80.5 \%$ \\
\hline \multicolumn{2}{|l|}{ Other } & \multicolumn{2}{|l|}{151} & $18.5 \%$ \\
\hline \multicolumn{2}{|l|}{ Missing } & \multicolumn{2}{|l|}{8} & $1.0 \%$ \\
\hline \multicolumn{2}{|l|}{ Total } & \multicolumn{2}{|l|}{817} & $100 \%$ \\
\hline \multicolumn{5}{|l|}{ Education } \\
\hline \multicolumn{2}{|l|}{ Primary } & \multicolumn{2}{|l|}{225} & $27.5 \%$ \\
\hline \multicolumn{2}{|l|}{ Secondary } & \multicolumn{2}{|l|}{342} & $41.9 \%$ \\
\hline \multicolumn{2}{|l|}{ Third level } & \multicolumn{2}{|l|}{223} & $27.3 \%$ \\
\hline Missing & & 27 & & $3.3 \%$ \\
\hline Total & & 817 & & $100 \%$ \\
\hline Employment status at $d$ & agnosis & & & \\
\hline $\begin{array}{l}\text { Employed/ } \\
\text { self-employed }\end{array}$ & & 369 & & $45.2 \%$ \\
\hline Other & & 399 & & $48.8 \%$ \\
\hline Missing & & 49 & & $6.0 \%$ \\
\hline Total & & 817 & & $100 \%$ \\
\hline Disease extent at diagn & & & & \\
\hline Stage $1 / 2$ & & 633 & & $77.5 \%$ \\
\hline Stage $3 / 4$ & & 144 & & $17.6 \%$ \\
\hline Missing & & 40 & & $4.9 \%$ \\
\hline Total & & 817 & & $100 \%$ \\
\hline Primary treatment(s) & & & & \\
\hline $\mathrm{RP}$ & & 258 & & $31.6 \%$ \\
\hline ERBT with ADT & & 156 & & $19.1 \%$ \\
\hline ERBT without ADT & & 253 & & $31.0 \%$ \\
\hline BT & & 65 & & $8.0 \%$ \\
\hline ADT only & & 44 & & $5.4 \%$ \\
\hline Monitoring only & & 27 & & $3.3 \%$ \\
\hline Missing & & 14 & & $1.7 \%$ \\
\hline Total & & 817 & & $100 \%$ \\
\hline Expectations of side eff & & & & \\
\hline $\begin{array}{l}\text { Did not have } \\
\text { side effects }\end{array}$ & & 123 & & $15.1 \%$ \\
\hline Not as bad as expect & & 195 & & $23.9 \%$ \\
\hline Same as expected & & 243 & & $29.7 \%$ \\
\hline Worse than expected & & 200 & & $24.5 \%$ \\
\hline Missing & & 56 & & $6.9 \%$ \\
\hline Total & & 817 & & $100 \%$ \\
\hline $\begin{array}{l}\text { Continuous } \\
\text { variables }\end{array}$ & Mean & SD & Range & $\begin{array}{l}\text { Possible } \\
\text { range }\end{array}$ \\
\hline Survivor age & 68.48 & 7.87 & $47-91$ & $18+$ \\
\hline $\begin{array}{l}\text { Time since diagnosis } \\
\text { (years) }\end{array}$ & 3.41 & 1.13 & $2-5$ & $2-5$ \\
\hline $\begin{array}{l}\text { Number of } \\
\text { comorbidities }\end{array}$ & 0.87 & 0.949 & $0-6$ & $0+$ \\
\hline $\begin{array}{l}\text { Number of side } \\
\text { effects }\end{array}$ & 2.16 & 1.72 & $0-8$ & $0-8$ \\
\hline FOR & 29.42 & 20.97 & $0-100$ & $0-100$ \\
\hline QOL & 86.56 & 14.35 & $12.39-100$ & $0-100$ \\
\hline
\end{tabular}

Abbreviations: ADT, androgen deprivation therapy; $\mathrm{BT}$, brachytherapy; ERBT, external beam radiotherapy; RP, radical prostatectomy. 
completed secondary education (42\%). Just under half reported working at diagnosis. In terms of health status, $78 \%$ has stage I/II cancer. The most common treatment type was RP (32\%) followed by ERBT without concurrent ADT (31\%). The mean number of comorbidities was 0.87 , although $44 \%$ reported none. On average, respondents reported having 2 ongoing treatment-related side effects; 19\% had none. In terms of retrospective appraisals, 30\% experienced side effects the same as expected, $24 \%$ reported side effects were not as bad as expected, and for $25 \%$ side effects were worse than expected. While FOR was low on average, there was considerable variation, suggesting that a sizeable minority experience considerable cancer-related worry with $19 \%$ having a FOR score above 50 (mean $=29.42$; standard deviation $=20.97$ ). QOL was generally high with $86 \%$ of the sample experiencing QOL scores in the top quartile (mean $=86.56$; standard deviation = 14.35)

\section{2 | Regression analysis}

The results of the regression analysis are displayed in Table 2 . Sociodemographic, health, and treatment-related factors (block 1) explained $28 \%$ of the variance in QOL scores $(P<0.001)$. The psychological appraisals (block 2) explained a further $9 \%$ of variance in QOL scores $(P<.001)$. The model as a whole was significant $(P<.001)$ and explained $37 \%$ of variance in QOL.

Consistent with our hypotheses both FOR $(\beta=-.29, P<.001)$ and expectations of side effects $(\beta=-.10, P=.002)$ were significant predictors of QOL, after adjusting for other factors. FOR was the strongest predictor of all the variables included in the model. Specifically, the higher the survivors' FOR, and the worse their experience of side effects compared with previous expectations, the lower their QOL.
Additional significant predictors of higher QOL were having fewer ongoing side effects ( $\beta=-.27, P<.001$ ), having fewer comorbid conditions $(\beta=-.21, P<.001)$, having less invasive treatment $(\beta=-.11$, $P=.001)$, having earlier stage cancer $(\beta=-.07, P=.02)$, and being a shorter time since diagnosis $(\beta=-.09, P=006)$. Two sociodemographic factors were significantly associated with higher QOL, specifically having achieved a higher level of education $(\beta=-.09, P=.002)$, and working at the time of diagnosis $(\beta=.10, P=.005)$.

\section{4 | DISCUSSION}

The aim of this study was to investigate the association between retrospective and prospective appraisals and QOL in prostate cancer survivors. Both these appraisals were associated with survivor well-being and were evident after accounting for a range of sociodemographic, health, and treatment-related predictors of QOL. This points to their importance and paves the way for interventions with a view to increasing QOL.

\subsection{The importance of appraisals and expectations}

The finding that expectations regarding future disease course were related to survivor QOL is in keeping with a number of studies which have shown the important influence FOR has in determining QOL in prostate cancer. ${ }^{14-17}$ We have shown that FOR acts independently of clinical variables such as cancer stage, number of comorbidities, treatment type, and side effects, in predicting QOL. The fact that FOR emerged overall as the strongest correlate of well-being clearly illustrates the pertinent relationship between prospective illness appraisals and well-being.

TABLE 2 Regression analyses for variables predicting QOL

\begin{tabular}{|c|c|c|c|c|c|c|c|}
\hline Variables & $\beta$ & $p$ & $t$ & B & SE & Cl95\% & \\
\hline \multicolumn{8}{|l|}{ Step 1: Sociodemographic, health, and treatment } \\
\hline Age & .023 & .540 & .613 & .046 & .076 & -.102 & .195 \\
\hline Marital status [other $=0 ;$ married/cohabiting $=1$ ] & .044 & .135 & 1.497 & 1.875 & 1.252 & -.584 & 4.333 \\
\hline Education level [higher = higher level of education] & $.092^{* *}$ & .002 & 3.087 & 1.820 & .590 & .663 & 2.978 \\
\hline Employment status [other = 0; employed $=1$ ] & $.095^{* *}$ & .005 & 2.803 & 3.012 & 1.075 & .902 & 5.122 \\
\hline Comorbidity [higher = more pre-existing conditions] & $-.208^{* * *}$ & .000 & -6.851 & -3.384 & .494 & -4.354 & -2.414 \\
\hline Time since diagnosis in years [higher = greater time] & $-.091^{* *}$ & .006 & -2.779 & -.406 & .146 & -.692 & -.119 \\
\hline Stage of cancer [stage $1 / 2=0 ;$ stage $3 / 4=1$ ] & $-.071^{*}$ & .018 & -2.370 & -2.763 & 1.166 & -5.053 & -.474 \\
\hline Primary treatment [higher = less invasive treatment] & $-.106^{* *}$ & .001 & -3.253 & -1.075 & .331 & -1.724 & -.426 \\
\hline Current side effects [higher = more side effects] & $-.267^{* * *}$ & .000 & -8.168 & -2.306 & .282 & -2.860 & -1.751 \\
\hline \multicolumn{8}{|l|}{$R^{2}$ Change $=0.28$} \\
\hline \multicolumn{8}{|l|}{ Step 2: Psychological appraisals } \\
\hline $\begin{array}{l}\text { Expectations of side effects [higher }=\text { worse than } \\
\text { expected] }\end{array}$ & $-.102^{* *}$ & .002 & -3.180 & -1.564 & .492 & -2.530 & -.598 \\
\hline Fear of recurrence [higher $=$ greater fears] & $-.290^{* * *}$ & .000 & -9.249 & -.219 & .024 & -.265 & -.172 \\
\hline \multicolumn{8}{|l|}{$R^{2}$ Change $=0.09$} \\
\hline$R^{2}=0.37$ & & & & & & & \\
\hline
\end{tabular}

Statistical significance:

${ }^{*} P<.05$.

${ }^{* *} P<.01$.

${ }^{* * *} P<.001$. 
It is also noteworthy that in our analysis QOL was independently associated with survivors' retrospective appraisals of their prior treatment expectations. Specifically, QOL was lower in survivors who reported their side effects to be worse than previously expected. This suggests that it is not only the severity or burden of side effects experienced which may influence QOL, ${ }^{4}$ but also how these experiences are interpreted by survivors in light of previous expectations. This is similar to our findings in relation to FOR, in that interpretations of risk, rather than actual clinical risk of recurrence, may be a stronger influence on QOL.

In keeping with these results, a number of longitudinal studies have examined how prospective patient expectations are related to later experience of side effects and QOL in cancer survivors. Often referred to as response expectancies, these have been shown to have associations with actual experience of side effects in that, when certain side effects are expected, they are more likely to be later reported. ${ }^{20,21,29,30}$ This has been termed the "nocebo" effect and is considered a neurobiological problem influenced by the way in which information is presented to patients. ${ }^{31}$ The nocebo effect has also been demonstrated in prostate cancer ${ }^{32}$ where pre-treatment expectations were associated with QOL following RP or radiotherapy.

Perhaps contrary to this research, our findings suggest that expectations of more positive outcomes prior to treatment were negatively associated with well-being when these expectations did not match actual experience. However, expectations were evaluated retrospectively rather than prospectively which may explain this differing effect. This finding fits with the wider literature on reasoning. ${ }^{24}$ More generally, this work suggests that when expectations are disconfirmed, especially in light of negative outcomes, individuals may experience surprise leading to a state of cognitive disequilibrium. ${ }^{33}$ Our findings suggest that when survivors experience unanticipated adverse side effects, this disequilibrium may lead to lower QOL, perhaps in a similar way in which QOL may be impacted by FOR, given that this represents perceived uncertainty over future disease course. On the other hand, it is interesting to note that those survivors who had more positive experiences than previously expected reported higher QOL. This suggests that expectation violation per se does not lead to lower QOL, rather it is how survivors interpret these expectation violations, either positively or negatively, that is important.

While more than half of survivors in our sample reported experiencing side effects that were either the same, or not as bad, as expected, the fact that approximately $25 \%$ reported worse side effects than expected suggests that a subset of survivors were not appropriately prepared for the effects of treatment. This fits with research reporting that prostate cancer patients can have unrealistic expectations regarding functioning after prostatectomy, ${ }^{22}$ although this may vary depending on the particular side effects involved or treatment undergone. For example, while only $22 \%$ experienced worse than expected bowel and hormonal functioning following surgery, almost half of patients experienced higher levels of urinary incontinence and worse sexual function than expected. ${ }^{22}$ Our results highlight the importance of acknowledging the role survivors' expectations have in their well-being. While providing patients with realistic expectations regarding treatment is desirable, our findings suggest there may be individual differences in the way survivors interpret these expectations retrospectively. Health care professionals should thus be sensitive to the way in which information on treatment and potential side effects is presented to patients so that $\mathrm{QOL}$ can remain high after treatment has ceased.

\section{2 | Other predictors of QOL}

Consistent with existing literature, ${ }^{34}$ it is not surprising to find that other sociodemographic, health, and treatment-related factors were related to higher QOL, including higher education, being in employment at diagnosis, being diagnosed at an earlier stage of the disease, and having fewer comorbid conditions. Interestingly, QOL decreased between 2 and 5 years post diagnosis, highlighting the complex time course of QOL in prostate cancer. ${ }^{35}$ This may be attributed to a greater number of new comorbidities due to ageing, or more complex psychological factors whereby men, once overcoming the initial shock of diagnosis, realise that their level of functioning may not recover. This fits with the finding that prostate cancer survivors have a lower QOL than other cancer survivors 10 years post diagnosis, suggesting that long-term side effects can persist in this group. ${ }^{35}$ Furthermore, we found survivors experiencing treatments such as ADT were more likely to experience lower QOL highlighting the detrimental impact this treatment can have on survivor well-being. ${ }^{5}$ These findings add to literature that highlights those most at risk of lower QOL in prostate cancer. Furthermore, utilising the summary score of the QLQC30 ${ }^{26}$ offers an alternative approach for measuring QOL that has not been extensively adopted in this population.

\section{3 | Study limitations}

It is important to acknowledge the limitations of this study. Firstly, given the cross-sectional design, we cannot assume causality or indeed the direction of the relationship between psychological appraisals and QOL. It is possible for example that men who have lower QOL were more likely to perceive their side effects to be worse than expected. As previously mentioned, our measure of expectations was based only on retrospective reflections rather than tracked longitudinally prior to treatment. Also, we only examined 2 aspects of survivors' illness appraisals. It is likely that other psychological appraisals of illness, both prospective and retrospective, may influence well-being. We also did not take a measure of current comorbidities (beyond those at diagnosis) which may have influenced results. Finally, our study focused only on survivors between 2 and 5 years post diagnosis so it is unclear how appraisals may influence QOL in longer term survivors.

\section{4 | Clinical implications}

Given that FOR is a common concern in prostate cancer, ${ }^{17}$ in spite of good prognosis following treatment, it is important health care professionals have a greater understanding of factors that put survivors most at risk of lower well-being. Our previous work in this study population found that FOR is associated with type of treatment and regret over treatment decisions. ${ }^{18}$ This suggests that simple measures, such as providing survivors with more information surrounding treatment, ${ }^{36,37}$ may be beneficial in decreasing FOR. As suggested by our current findings, such interventions might also yield benefits in terms of QOL. 
Consistent with recommendations, ${ }^{32}$ our findings highlight the importance of ensuring patients have appropriate expectations regarding QOL following treatment of prostate cancer. Health care professionals should be sensitive to the way in which information regarding what to expect from treatment is presented to patients and, more generally, should strive to support patients prior to, during, and after treatment in order to facilitate positive appraisals throughout the survivorship process.

\section{5 | CONCLUSIONS}

Health care professionals now recognise the significant effect QOL has on survivor prognosis and, as such, acknowledge the importance in taking measures to improve this. Our study has shown that psychological appraisals, both retrospective (in terms of mismatched side-effect expectations and experiences) and prospective (in terms of FOR), are strongly related to QOL. This suggests that interventions focused on alleviating fears and considering the most appropriate ways to present information regarding potential side effects of treatment offer potential to improve well-being among cancer survivors.

\section{ACKNOWLEDGEMENTS}

We would like to thank the men who took part in this study and the health professionals who helped screen men for eligibility. We would also like to thank Dr Heather Kinnear and Dr Sandra Deady. The funders had no role in the conduct of the research, determining the content of the paper, or writing of the manuscript. This work was supported by the Health Research Board in the Republic of Ireland (HRA_HSR/2010/17), Prostate Cancer UK (NI09-03 and NI-PG13001), the R\&D office of the Public Health Agency in Northern Ireland, and the National Cancer Control Programme and received no specific grant from any funding agency in the commercial or not-for-profit sectors. The Northern Ireland Cancer Registry is funded by the Public Health Agency for Northern Ireland and the National Cancer Registry Ireland by the Department of Health.

\section{ORCID}

Rebecca Maguire (D) http://orcid.org/0000-0002-0368-4626

Paul Hanly (i) http://orcid.org/0000-0002-5348-4825

Frances J. Drummond (D) http://orcid.org/0000-0002-7802-776X

Anna Gavin (D) http://orcid.org/0000-0002-5858-928X

Linda Sharp (D) http://orcid.org/0000-0001-9515-1722

\section{REFERENCES}

1. Aaronson NK, Ahmedzai S, Bergman B, et al. The European Organization for Research and Treatment of Cancer QLQ-C30: a quality-of-life instrument for use in international clinical trials in oncology. J Natl Cancer Inst. 1993;85(5):365-376.

2. Siegel RL, Miller KD, Jemal A. Cancer statistics, 2015. CA Cancer J Clin. 2015;65(1):5-29.

3. Sanda MG, Dunn RL, Michalski J, et al. Quality of life and satisfaction with outcome among prostate-cancer survivors. New England Journal of Medicine. 2008:358(12):1250-1261.

4. Gavin AT, Drummond FJ, Donnelly C, O'Leary E, Sharp L, Kinnear HR. Patient-reported 'ever had' and 'current' long-term physical symptoms after prostate cancer treatments. BJU Int. 2015;116(3):397-406.
5. Drummond FJ, Kinnear H, O'Leary E, Gavin A, Sharp L. Long-term health-related quality of life of prostate cancer survivors varies by primary treatment. Results from the PiCTure (Prostate Cancer Treatment, your experience) study. J Cancer Surviv. 2015;9(2):361-372.

6. Ferrer M, Guedea F, Suárez JF, et al. Quality of life impact of treatments for localized prostate cancer: cohort study with a 5year follow-up. Radiother Oncol. 2013;108(2):306-313.

7. Sharp L, O'Leary E, Kinnear H, Gavin A, Drummond FJ. Cancer-related symptoms predict psychological wellbeing among prostate cancer survivors: results from the PiCTure study. Psychooncology. 2016;25(3):282-291.

8. Eton DT, Lepore SJ. Prostate cancer and health-related quality of life: a review of the literature. Psychooncology. 2002;11(4):307-326.

9. de la Torre-Luque A, Gambara H, López E, Cruzado JA. Psychological treatments to improve quality of life in cancer contexts: a meta-analysis. International Journal of Clinical and Health Psychology. 2016;16(2):211-219.

10. Osborn RL, Demoncada AC, Feuerstein M. Psychosocial interventions for depression, anxiety, and quality of life in cancer survivors: meta-analyses. The International Journal of Psychiatry in Medicine. 2006;36(1):13-34.

11. Crist JV, Grunfeld EA. Factors reported to influence fear of recurrence in cancer patients: a systematic review. Psychooncology. 2013;22(5):978-986.

12. Simard S, Thewes B, Humphris $G$, et al. Fear of cancer recurrence in adult cancer survivors: a systematic review of quantitative studies. $J$ Cancer Surviv. 2013;7(3):300-322.

13. Simard S, Savard J, Ivers H. Fear of cancer recurrence: specific profiles and nature of intrusive thoughts. J Cancer Surviv. 2010;4(4):361-371.

14. Bellizzi KM, Latini DM, Cowan JE, DuChane J, Carroll PR. Fear of recurrence, symptom burden, and health-related quality of life in men with prostate cancer. Urology. 2008;72(6):1269-1273.

15. Hart SL, Latini DM, Cowan JE, Carroll PR, CaPSURE ${ }^{T M}$ Investigators. Fear of recurrence, treatment satisfaction, and quality of life after radical prostatectomy for prostate cancer. Support Care Cancer. 2008;16(2):161-169.

16. Mehta SS, Lubeck DP, Pasta DJ, Litwin MS. Fear of cancer recurrence in patients undergoing definitive treatment for prostate cancer: results from CaPSURE. J Urol. 2003;170(5):1931-1933.

17. van de Wal M, van Oort I, Schouten J, Thewes B, Gielissen M, Prins J. Fear of cancer recurrence in prostate cancer survivors. Acta Oncol. 2016;1-7.

18. Maguire R, Hanly P, Balfe M, et al. Worry in head and neck cancer caregivers: the role of survivor factors, care-related stressors, and loneliness in predicting fear of recurrence. Nurs Res. 2017;66(4):295-303.

19. Diefenbach MA, Mohamed NE. Regret of treatment decision and its association with disease-specific quality of life following prostate cancer treatment. Cancer Invest. 2007;25(6):449-457.

20. Sohl SJ, Schnur JB, Montgomery GH. A meta-analysis of the relationship between response expectancies and cancer treatment-related side effects. J Pain Symptom Manage. 2009;38(5):775-784.

21. Devlin EJ, Denson LA, Whitford HS. Cancer treatment side effects: a meta-analysis of the relationship between response expectancies and experience. J Pain Symptom Manage. 2017;54(2):245-258.e2.

22. Wittmann D, He C, Coelho M, Hollenbeck B, Montie JE, Wood DP. Patient preoperative expectations of urinary, bowel, hormonal and sexual functioning do not match actual outcomes 1 year after radical prostatectomy. J Urol. 2011;186(2):494-499.

23. Coyle C, Morgan E, Drummond FJ, Sharp L, Gavin A. Do men regret prostate biopsy: results from the PiCTure study. BMC Urol. 2017;17(1):11.

24. Burgoon JK, Hale JL. Nonverbal expectancy violations: model elaboration and application to immediacy behaviors. Communications Monographs. 1988;55(1):58-79. 
25. Drummond FJ, Kinnear H, Donnelly C, et al. Establishing a populationbased patient-reported outcomes study (PROMs) using national cancer registries across two jurisdictions: the Prostate Cancer Treatment, your experience (PiCTure) study. BMJ Open. 2015;5(4):e006851.

26. Giesinger JM, Kieffer JM, Fayers PM, et al. Replication and validation of higher order models demonstrated that a summary score for the EORTC QLQ-C30 is robust. J Clin Epidemiol. 2016;69:79-88.

27. Gray NM, Sharp L, Cotton SC, et al. Developing a questionnaire to measure the psychosocial impact of an abnormal cervical smear result and its subsequent management: the TOMBOLA (Trial Of Management of Borderline and Other Low-grade Abnormal smears) trial. Qual Life Res. 2005;14(6):1553-1562.

28. Greenberg DB, Kornblith AB, Herndon JE, et al. Quality of life for adult leukemia survivors treated on clinical trials of cancer and leukemia group B during the period 1971-1988. Cancer. 1997;80(10):1936-1944.

29. Olver IN, Taylor AE, Whitford HS. Relationships between patients' pre-treatment expectations of toxicities and post chemotherapy experiences. Psychooncology. 2005;14(1):25-33.

30. Nestoriuc $\mathrm{Y}$, von Blanckenburg $\mathrm{P}$, Schuricht $\mathrm{F}$, et al. Is it best to expect the worst? Influence of patients' side-effect expectations on endocrine treatment outcome in a 2-year prospective clinical cohort study. Ann Oncol. 2016;27(10):1909-1915.

31. Colloca L, Miller FG. The nocebo effect and its relevance for clinical practice. Psychosom Med. 2011;73(7):598-603.

32. Symon Z, Daignault S, Symon R, Dunn RL, Sanda MG, Sandler HM. Measuring patients' expectations regarding health-related quality-oflife outcomes associated with prostate cancer surgery or radiotherapy. Urology. 2006;68(6):1224-1229.
33. Maguire R, Maguire P, Keane MT. Making sense of surprise: an investigation of the factors influencing surprise judgments. J Exp Psychol Learn Mem Cogn. 2011;37(1):176-186.

34. Litwin MS, Hays RD, Fink A, et al. Quality-of-life outcomes in men treated for localized prostate cancer. JAMA. 1995;273(2):129-135.

35. Wang SY, Hsu SH, Gross CP, et al. Association between time since cancer diagnosis and health-related quality of life: a population-level analysis. Value Health. 2016;19(5):631-638.

36. Adsul P, Wray R, Spradling K, Darwish O, Weaver N, Siddiqui S. Systematic review of decision aids for newly diagnosed patients with prostate cancer making treatment decisions. J Urol. 2015;194(5):1247-1252.

37. Stacey D, Légaré F, Lewis K, et al. Decision aids for people facing health treatment or screening decisions. Cochrane Libr. 2017.

\section{SUPPORTING INFORMATION}

Additional Supporting Information may be found online in the supporting information tab for this article.

How to cite this article: Maguire R, Hanly P, Drummond FJ, Gavin A, Sharp L. Expecting the worst? The relationship between retrospective and prospective appraisals of illness on quality of life in prostate cancer survivors. Psycho-Oncology. 2018;27:1237-1243. https://doi.org/10.1002/pon.4660 